\title{
Backstepping Control for Nonlinear Dual Smart Drive
}

\author{
Roemi Fernández*, João Hespanha ${ }^{\dagger}$, Teodor Akinfiev ${ }^{\ddagger}$ and Manuel Armada
}

\begin{abstract}
Intended for being used in legged robots, a specially designed nonlinear actuator, Dual Smart Drive, which offers continuously changing transmission ratio and dual properties, and that is very suitable for situations where the same drive is required to perform two different types of start-stop motions of the mobile link, is introduced. Then, the associated control problem to this nonlinear actuator is established and a backstepping design strategy is adopted to develop a Lyapunov-based nonlinear controller that ensures asymptotic tracking of the desired laws of motion, which have been properly selected using time optimal control. Finally, experimental results are presented to show the effectiveness and feasibility of the proposed nonlinear control method for the Dual Smart Drive.
\end{abstract}

\section{INTRODUCTION}

Presently legged robots performance is characterised both by very low speeds and high energy expense, resulting in low efficiency for these machines. This feature, due mainly to their actuation being realised with conventional drives, restricts notably their potential [1] and limits the time of autonomous robot operation. In this respect the selection of appropriated drive systems is one of the most determining factors to overcome the stated drawbacks [2], [3]. Several authors [4], [5], [6] have demonstrated that the employment of quasi-resonance drives instead of conventional ones represents a good way for increasing the robot efficiency. They use variable geometry to accomplish variable reduction and the arrangement gives different transmission ratios at different positions of the output joint.

The drive described here, the Dual Smart [7], is also a further development of the quasi-resonance drives and it provides a continuously changing transmission ratio according to the angular position of the mobile link. Its nonlinear transmission ratio varies smoothly from a minimum value at the middle position of the mobile link, ad infinitum value in the extreme positions of the mobile link. Nevertheless, this drive has the additional advantage of presenting two possible magnitudes of the reduction ratio for each position of the mobile link due to the particular configuration of the mechanism linkages. This dual property

\footnotetext{
*Supported by the Spanish Ministry of Education under Grant F.P.U.

'Supported by the National Science Foundation under Grant No. ECS0242798 .

${ }^{+}$Supported by the Spanish Ministry of Science and Technology under Grant Ramón y Cajal.

R. Fernández, T. Akinfiev and M. Armada are with the Industrial Automation Institute, Spanish Council for Scientific Research, La Poveda 28500 Arganda del Rey, Madrid, Spain roemi@iai.csic.es, teodor@iai.csic.es, armada@iai.csic.es

J. Hespanha is with the Center for Control Engineering and Computation, University of California, Santa Barbara, CA 93106-9560 USA hespanha@ece.ucsb.edu
}

permits to arrange the linkage mechanism within the limits of one angle, when the load (or the external force) is small (and to gain for high speeds of displacement), or within the limits of another angle, when the load is greater (having, correspondingly, smaller displacement velocities). Consequently, the utilisation of this drive allows to increase the motion speed of walking machines by enabling high acceleration and deceleration at the beginning and at the end of the driving (high absolute magnitude of the reduction ratio), and for maintaining high speeds in the intermediate part of the driving trajectory both for legs and body (low absolute magnitude of the reduction ratio).

The use of such a kind of nonlinear actuator augments control system complexity. In order to solve that problem, a combined backstepping/time-optimal control strategy is proposed. The basic idea is to use the backstepping design technique to develop a Lyapunov-based nonlinear controller for the Dual Smart Drive that achieves asymptotic tracking of the reference trajectories, which have been suitable selected using time optimal control method [8].

The rest of the paper is organized as follows. Section II describes the Dual Smart Drive and its nonlinear mathematical model. Section III is devoted to the time optimal control problem for the calculation of the reference trajectories. Section IV explains the nonlinear controller design using backstepping technique. The performance of the resulting controller is validated in section $\mathrm{V}$ through experimental testing. Finally section VI summarises major conclusions.

\section{SYSTEM DESCRIPTION}

The design of the actuator was presented in [9]. It consists of a crank connected to the reducing gear of a DC motor, a mobile link that rotates around a fixed point, and a slider that slips along the mobile link in a radial direction thanks to the movement of the crank to which it is connected. An essential characteristic of this actuator is that the length of the crank is smaller than the distance between the mobile link rotation axis and the crank rotation axis (see Fig. 1). The kinematics is determined by the following parameters: $\ell_{M L}$ - distance between the mobile link rotation axis and the crank rotation axis, $\ell_{C}$ - length of the crank, $\varphi$ - angular position of the rotor measured clockwise from the $o x$ axis, $\alpha$ - angular position of the crank measured clockwise from the $o x$ axis, and $\beta$ angular position of the mobile link measured clockwise from the $o x$ axis. Taking into account that $\ell_{C}<\ell_{M L}$, the mobile link will move between two extreme positions (see Fig. 1):

$$
-\beta_{0}<\beta<\beta_{0} \quad \text { where } \quad \beta_{0}=\arcsin \left(\frac{\ell_{C}}{\ell_{M L}}\right) .
$$


A displacement of the mobile link from one end position to the other one can be carried out in two different ways by displacement of the crank within the limits of the angle $\Gamma_{1}$ (so-called first regime) or within the limits of the angle $\Gamma_{2}$ (so-called second regime). As the relationship between the angular position of the rotor and the angular position of the crank is $\varphi=K_{G} \alpha,\left(K_{G}\right.$ - constant transmission of the reduction gear), the variation of the angular position of the mobile link $\beta$ as function of the angular position of the rotor $\varphi$ is given by:

$$
\beta=\operatorname{arctg}\left(\frac{\operatorname{Sin}\left(\varphi / K_{G}\right)}{\left(\ell_{M L} / \ell_{C}\right)+\operatorname{Cos}\left(\varphi / K_{G}\right)}\right) .
$$

The nonlinear transmission ratio $K_{D}$ between the reducing
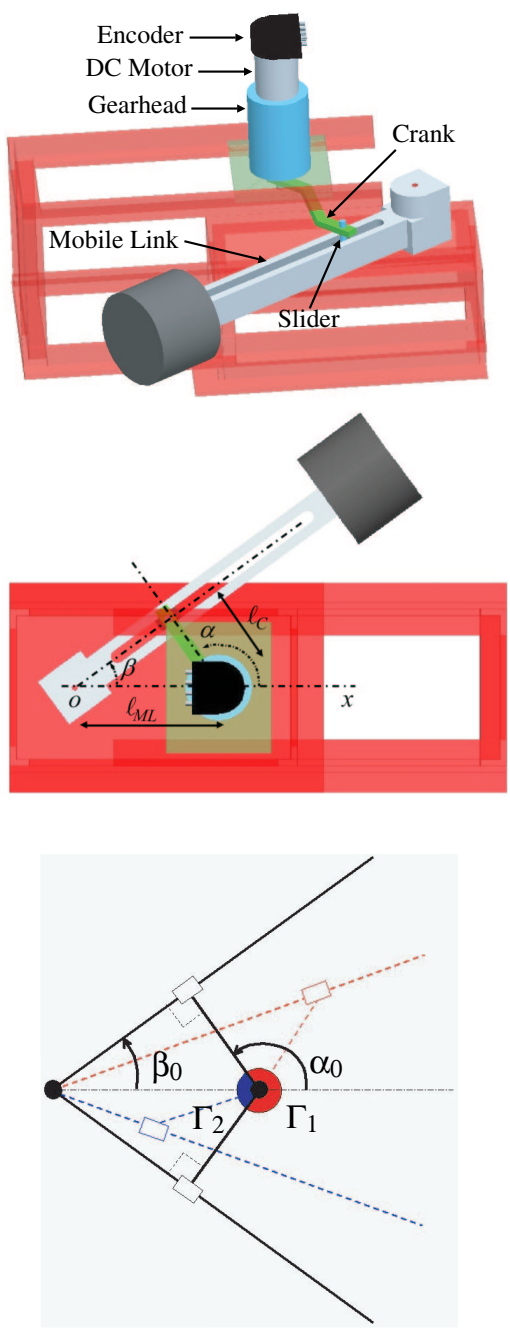

Fig. 1. Elements and kinematical schema of the Dual Smart Drive.

gear and the mobile link can be calculated using $K_{D}=\dot{\alpha} / \dot{\beta}$, where $\dot{\alpha}$ is the angular velocity of the crank and $\dot{\beta}$ is the angular velocity of the mobile link. As a result,

$$
K_{D}=\frac{1+\left(\ell_{M L} / \ell_{C}\right)^{2}+2\left(\ell_{M L} / \ell_{C}\right) \operatorname{Cos} \alpha}{1+\left(\ell_{M L} / \ell_{C}\right) \operatorname{Cos} \alpha} .
$$

This last equation has a $2 \pi$ periodic character consisting in two different parts (see Fig. 3): one with negative values

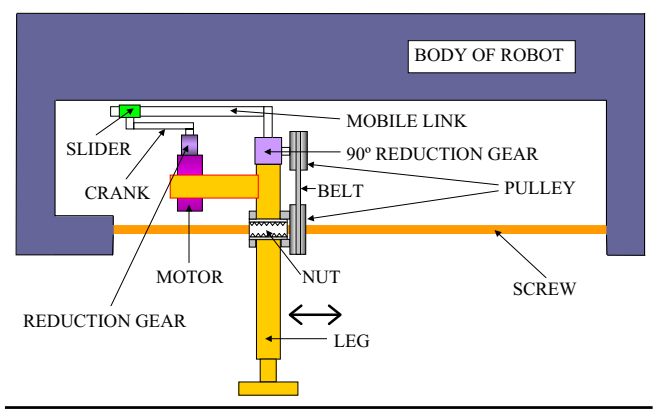

Fig. 2. Example of Dual Smart Drive connection on a legged robot.

(part $\Gamma_{2}$ ), and another one with positive values (part $\Gamma_{1}$ ). The negative magnitude of the reduction ratio means that the crank and the mobile link are rotating in opposite directions. It is interesting to notice that the reduction ratio tends to infinity at the end points, $-\beta_{0}, \beta_{0}$, where the crank is perpendicular to the mobile link. In these points, the deviation of the mobile link from its medium position is maximal. That is why, the movement of the mobile link from one end position to the other one ensures the most favourable change of the reduction ratio for maintaining high accelerations at the beginning and at the end of driving (high absolute magnitude of the reduction ratio) and for maintaining high speeds in the intermediate part of the trajectory (low absolute magnitude of the reduction ratio). Moreover, when the motion of the mobile link is carried out by displacement of the crank within the limits of the angle $\Gamma_{1}$, the absolute average magnitude of the reduction ratio will be greater than when displacement is within the limits of the angle $\Gamma_{2}$ (see Fig. 4). This enables to use the movement of the crank within the limits of the angle $\Gamma_{2}$ when the load is small (i.e. for the motion of the robot's leg) and to gain high speeds of displacement, or within the limits of the angle $\Gamma_{1}$ when the load is great (i.e. for the motion of the robot's body), having correspondingly, smaller velocities of displacement. Therefore, the drive allows easily to perform a displacement of the mobile link from one end position to another and to have two different laws for changing drive's reduction ratio [9]. It is important to point out that at the singular points, where the drive changes its working regime, the velocities are null. This essential feature gives us the possibility to model the Dual Drive dynamics independently for each working regime and to combine both of them for a global representation. Additionally, a horizontal operation of the drive is assumed in order to achieve a gravitational decoupling. Bearing these facts in mind, the mobile link equation is given by:

$$
J_{i} \ddot{\beta}_{i}=K_{D} K_{G} M_{i}-b_{i} \dot{\beta}_{i}-M_{F R i} \operatorname{sign}\left(\dot{\beta}_{i}\right),
$$

where $J_{i}$ is the equivalent inertia of the mobile link in each working regime $i=1,2, \ddot{\beta}_{i}$ is the angular acceleration of the 


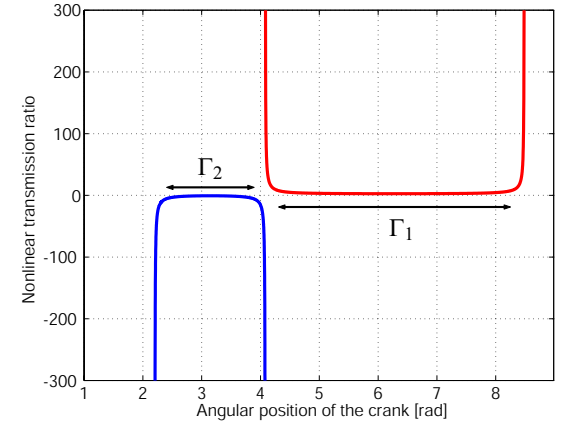

Fig. 3. Nonlinear transmission ratio as function of the angular position of the crank.

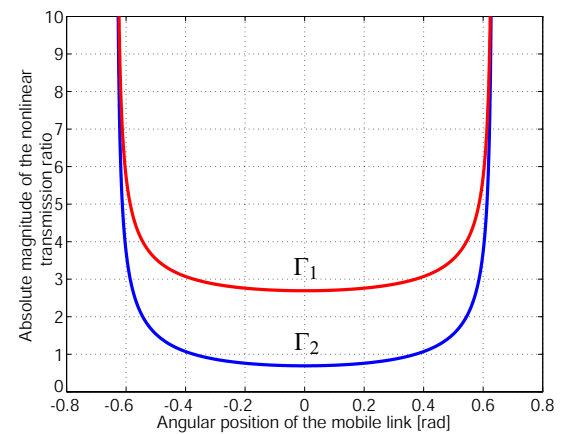

Fig. 4. Absolute magnitudes of the nonlinear transmission ratio as function of the angular position of the mobile link.

mobile link, $M_{i}$ is the moment that actuates over the mobile link, $b_{i}$ is the equivalent viscosity friction coefficient and $M_{F R i}$ is the moment of dry friction during the movement in each regime. The rotor equation is given by:

$$
J_{M} \ddot{\varphi}_{i}=\frac{k_{m}}{R_{M}}\left[u_{i}-k_{E} \dot{\varphi}_{i}\right]-M_{i}-b_{M} \dot{\varphi}_{i},
$$

where $J_{M}$ is the rotor inertia, $\ddot{\varphi}_{i}$ is the angular acceleration of the rotor, $k_{m}$ is the torque constant, $u_{i}$ is the input control motor terminal voltage, $R_{M}$ is the motor resistance, $k_{E}$ is the back - EMF constant, and $b_{M}$ is the viscosity friction coefficient on the motor shaft.

Combining all these equations the dynamic model of the system with a nonlinear transmission ratio results in:

$$
\begin{aligned}
& \dot{x}_{1 i}=x_{2 i}, \\
& \dot{x}_{2 i}=f\left(x_{1 i}, x_{2 i}\right)+K\left(x_{1 i}\right) u_{i} .
\end{aligned}
$$

where $x_{1 i}$ denotes the angular position of the rotor $\varphi, x_{2 i}$ the corresponding angular velocity $\dot{\varphi}$, and,

$$
\begin{aligned}
& f\left(x_{1 i}, x_{2 i}\right)=J_{D i\left(x_{1 i}\right)}\left[\left(\frac{J_{G i} \dot{K}_{D\left(x_{1}, x_{2}\right)}}{K_{D\left(x_{1}\right)}^{3}}-K_{M a}-\right.\right. \\
& \left.\left.-\frac{b_{G i}}{K_{D\left(x_{1}\right)}^{2}}\right) x_{2 i}-\frac{M_{F R G i}}{K_{D\left(x_{1}\right)}} \operatorname{sign}\left(\frac{x_{2 i}}{K_{D\left(x_{1}\right)} K_{G}}\right)\right],
\end{aligned}
$$

$$
\begin{aligned}
& K\left(x_{1 i}\right)=K_{M b} J_{D i\left(x_{1 i}\right)}, \\
& J_{G i}=\frac{J_{i}}{K_{G}^{2}}, \quad K_{M a}=b_{M}+\frac{k_{E} k_{m}}{R_{M}}, \\
& b_{G i}=\frac{b_{i}}{K_{G}^{2}}, \quad M_{F R G i}=\frac{M_{F R i}}{K_{G}}, \\
& K_{M b}=\frac{k_{m}}{R_{M}}, \quad J_{D i}\left(x_{1 i}\right)=\frac{K_{D\left(x_{1}\right)}^{2}}{J_{M} K_{D\left(x_{1}\right)}^{2}+J_{G i}} .
\end{aligned}
$$

\section{TIME OPTIMAL CONTROL}

In the previous section it has been argued that the movement from one end position to the other one ensures a favorable change of the reduction ratio for each working regime. Then, the desired control objective is to make this displacement in a minimum time using all the capabilities that the electromotor and the transmission have available. For this reason, a time optimal control [8] will be used for the calculation of the reference trajectories.

For nonlinear systems, optimal control theory only provides necessary conditions for optimality. Hence, only a set of candidate controls can be deduced using the general theory. So, once the equations of motion have been derived, the Pontryagin's Minimum Principle is applied to obtain the necessary conditions for optimality. Then, the equations for the state and co-state vector that satisfy the necessary conditions are determined and subsequently, the control sequences that can be candidates for time optimal control are obtained.

The control problem is to minimize the cost functional

$$
\Psi\left(u_{i}\right)=\int_{t_{0}}^{T} d t=T-t_{0}, \quad T \text { is free, }
$$

subject to a magnitude input constraint of the form $\left|u_{i}(t)\right| \leq$ $u_{\max }, \forall t \in\left[t_{0}, T\right]$. The Hamiltonian function for the system (6) and the cost functional (7) is given by:

$$
\begin{aligned}
H_{i}(x, p, u)= & 1+x_{2 i} p_{1 i}+f\left(x_{1 i}, x_{2 i}\right) p_{2 i}+ \\
& +K\left(x_{1 i}\right) u_{i} p_{2 i} .
\end{aligned}
$$

Since the Hamiltonian is linear on $u_{i}$, the optimal control is of the form:

$$
u_{i}^{*}(t)=u_{\max } \operatorname{sign}\left[K\left(x_{1 i}^{*}(t)\right) p_{2 i}^{*}(t)\right]
$$

almost everywhere on $\left[t_{0}, T^{*}\right]$, where $T^{*}$ is the minimum time, and $x_{1 i}^{*}(t)$ and $p_{2 i}^{*}(t)$ are the state and co-state trajectories under the optimal control law. Thus the time optimal control is bang-bang. This means that one can partition the state space into two regions, one on which $u_{i}=u_{\max }$ and another one on which $u_{i}=-u_{\max }$. The boundary between them is called the switching curve. For second-order systems such as this one, one can determine the switching curve by plotting system trajectories in the 


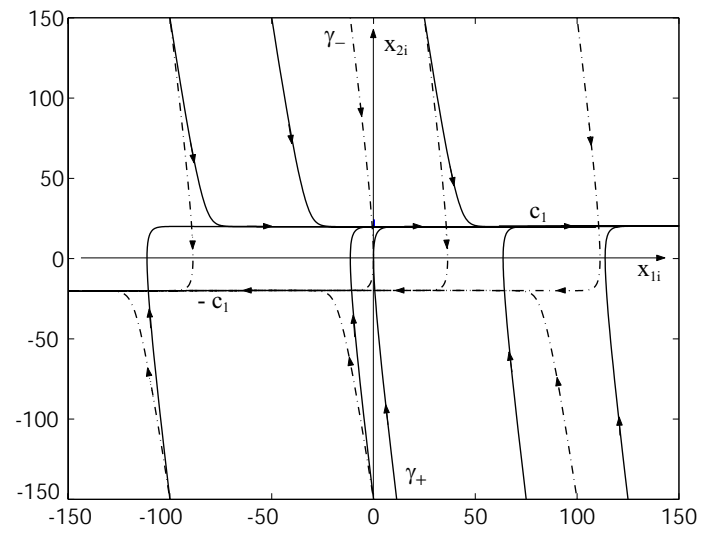

Fig. 5. State plane trajectories for the system given by (6). For illustration purposes we used $u_{\max }=1$.

phase plane for the two extreme control values. Fig. 5 shows trajectories of the system (6) for $u_{i}=u_{\max }$ (solid curves) and $u_{i}=-u_{\max }$ (dashed curves). The arrows indicate direction of motion of the state. One can see that all the trajectories due to $u_{i}=+u_{\max }$ tend to the line $x_{2 i}=c_{1}$ and all the trajectories due to $u_{i}=-u_{\max }$ tend to the line $x_{2 i}=$ $-c_{1}$. The trajectories which pass through the origin are labelled as $\gamma_{+}$and $\gamma_{-}$[8]. The $\gamma_{+}$curve is the locus of all points $\left(x_{1 i}, x_{2 i}\right)$ which can be forced to $(0,0)$ by the control $u_{i}=+u_{\max }$ and the $\gamma_{-}$curve is the locus of all points $\left(x_{1 i}, x_{2 i}\right)$ which can be forced to $(0,0)$ by the control $u_{i}=-u_{\max }$. The $\gamma$ curve, called the switching curve, is the union of the $\gamma_{+}$and $\gamma_{-}$curves and it divides the state plane into two regions $R_{+}$and $R_{-} . R_{+}$consists of the points to the left of the switching curve $\gamma$ and $R_{-}$consists of the points to the right of the switching curve $\gamma$ (cf. Fig. 6). As the bang-bang control has a finite number of switches on every bounded time interval, it shall be demonstrated that the extremal controls for the system (6) can switch at most once and that only the four control sequences $\left\{+u_{\max }\right\},\left\{-u_{\max }\right\}$, $\left\{+u_{\max },-u_{\max }\right\},\left\{-u_{\max },+u_{\max }\right\}$ can be candidates for time optimal control. The arguments are illustrated in Fig. 7. If the initial state $\Xi=\left(\xi_{1}, \xi_{2}\right)$ belongs to the $\gamma_{+}$, by definition, the control sequence $\left\{+u_{\max }\right\}$ results in the trajectory $\Xi 0$, which reaches the origin. The control sequence $\left\{-u_{\max }\right\}$ results in the trajectory $\Xi A^{\prime}$, which never reaches the origin. The control sequence $\left\{+u_{\max },-u_{\max }\right\}$ results in trajectories of the type $\Xi B^{\prime} C^{\prime}$, which never reach the origin. The control sequence $\left\{-u_{\max },+u_{\max }\right\}$ results in trajectories of the type $\Xi D^{\prime} E^{\prime}$, which never reach the origin. Therefore, if the initial state is on the $\gamma_{+}$curve, from all the control sequences which are candidates for minimum-time control, only the sequence $\left\{+u_{\max }\right\}$ can force the state $\Xi$ to 0 . Thus by elimination, it must be time optimal. Using analogous arguments, we can show that if the initial state belongs to the $\gamma_{-}$curve, then the time optimal control is $u_{i}=-u_{\max }$. Thus, the time optimal control law for initial states on the $\gamma$ curve has been derived. Let us now consider an initial state $X$ which belongs to the region $R_{+}$.

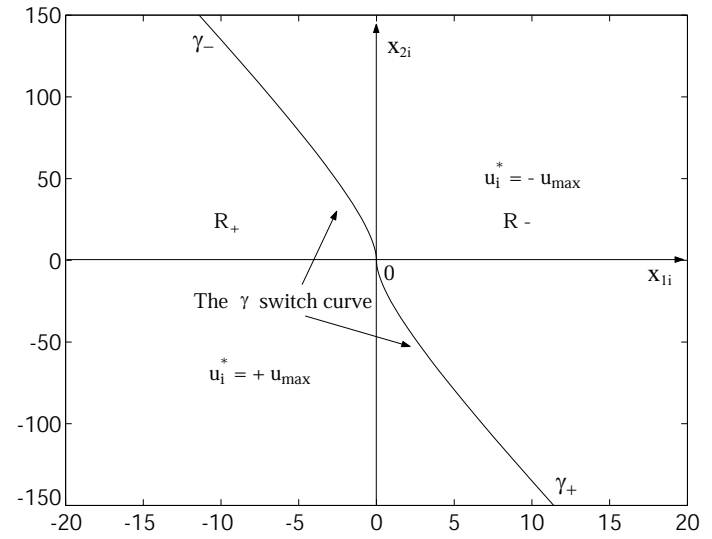

Fig. 6. Switching curve for the second order nonlinear system (6). For illustration purposes we used $u_{\max }=1$.

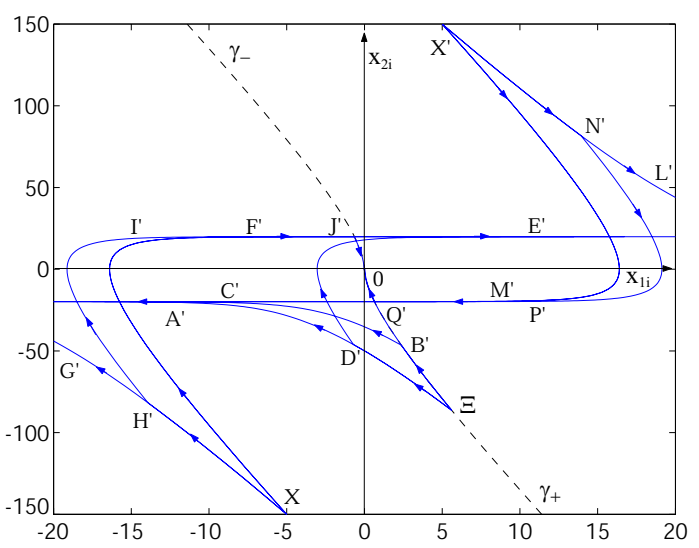

Fig. 7. Various trajectories generated by the four possible control sequences. For illustration purposes $u_{\max }=1$ was used.

If we use the control sequence $\left\{+u_{\max }\right\}$, the resulting trajectory is $X F^{\prime}$, shown in Fig. 7, which never reaches the origin. If the sequence $\left\{-u_{\max }\right\}$ is applied, the resulting trajectory $X G^{\prime}$ never reaches the origin. If we apply the sequence $\left\{-u_{\max },+u_{\max }\right\}$, the resulting trajectory is of the type $X H^{\prime} I^{\prime}$, which does not reach the origin. However, if we use the sequence $\left\{+u_{\max },-u_{\max }\right\}$, then one can reach the origin along the trajectory $X J^{\prime} 0$, provided that the transition from the control $u_{i}=+u_{\max }$ to $u_{i}=-u_{\max }$ occurs at the point $J^{\prime}$, that is, at the precise moment that the trajectory crosses the $\gamma$ switching curve. This is true for every state in $R_{+}$. Thus, by the process of elimination, we have arrived at the conclusion that the sequence $\left\{+u_{\max },-u_{\max }\right\}$ is time optimal for every state in $R_{+}$, provided that the control switches from $u_{i}=+u_{\max }$ to $u_{i}=-u_{\max }$ at the $\gamma$ switching curve. Using identical arguments one concludes that when the initial state belongs to $R_{-}$the sequence $\left\{-u_{\max },+u_{\max }\right\}$ is time optimal with the transition from $-u_{\max }$ to $u_{\max }$ over $\gamma$. The time optimal control $u_{i}^{*}$ can therefore be written as a function of the state as follows:

$$
\begin{array}{ll}
u_{i}^{*}\left(x_{1 i}, x_{2 i}\right)=+u_{\max } & \forall\left(x_{1 i}, x_{2 i}\right) \in \gamma_{+} \cup R_{+}, \\
u_{i}^{*}\left(x_{1 i}, x_{2 i}\right)=-u_{\max } & \forall\left(x_{1 i}, x_{2 i}\right) \in \gamma_{-} \cup R_{-} .
\end{array}
$$


Bang-bang control is useful for establishing a theoretical bound on the best possible controlled system performance, but its practical is quite difficult [10], [11]. Generally, its performance degrades severely with modelling inaccuracies, unpredicted external disturbances or measurement noise. To add stability, a combination of time optimal control and backstepping is proposed, by using the time optimal trajectories as reference values for a controller designed using integrator backstepping. Thus, the approaches will be quasi time optimal rather than exactly time optimal.

\section{BACKSTEPPING}

In order to solve the tracking problem, a nonlinear trajectory tracking controller is proposed following the integrator backstepping technique [12], [13], [14]. Firstly, a coordinate transformation is introduced for the system (6):

$$
\begin{aligned}
\dot{e}_{1 i}= & e_{2 i}+x_{d 2 i}-\dot{x}_{d 1 i}, \\
\dot{e}_{2 i}= & J_{D i}\left[\left(G_{D i}-K_{M a}\right)\left(e_{2 i}+x_{d 2 i}\right)-M_{Z i}\right]+ \\
& +K_{M b} J_{D i} u_{i}-\dot{x}_{d 2 i} .
\end{aligned}
$$

where $e_{1 i}=x_{1 i}-x_{d 1 i}, e_{2 i}=x_{2 i}-x_{d 2 i}$ denote the position and velocity tracking errors, $x_{d 1 i}=x_{1 i}^{*}$ and $x_{d 2 i}=x_{2 i}^{*}$, (for $\left.i=1,2\right)$ the time optimal trajectories determined in Section 3 and

$$
\begin{gathered}
G_{D i}=\frac{J_{G i} \dot{K}_{D\left(e_{1 i}+x_{d 1 i}, z_{2 i}+\dot{x}_{d 1 i}-k_{1 i} e_{1 i}\right)}-\frac{b_{G i}}{K_{D\left(e_{1 i}+x_{d 1 i}\right)}^{3}},}{K_{D\left(e_{1 i}+x_{d 1 i}\right)}^{2}} \\
M_{Z i}=\frac{M_{F R G i}}{K_{D\left(e_{1 i}+x_{d 1 i}\right)}} \operatorname{sign}\left(\frac{z_{2 i}+\dot{x}_{d 1 i}-k_{1 i} e_{1 i}}{K_{D\left(e_{1 i}+x_{d 1 i}\right)} K_{G}}\right) .
\end{gathered}
$$

Now, a smooth positive definite Lyapunov-like function is defined:

$$
V_{1 i}=\frac{1}{2} e_{1 i}^{2}
$$

Its derivative is given by:

$$
\dot{V}_{1 i}=e_{1 i}\left(e_{2 i}+x_{d 2 i}-\dot{x}_{d 1 i}\right) \text {. }
$$

Next, $e_{2 i}$ is regarded as a virtual control law to make $\dot{V}_{1 i}$ negative. This is achieved by setting $e_{2 i}$ equal to $-x_{d 2 i}+$ $\dot{x}_{d 1 i}-k_{1 i} e_{1 i}$, for some positive constant $k_{1 i}$. To accomplish this, an error variable $z_{2 i}$ that we would like to set to zero is introduced:

$$
z_{2 i}=e_{2 i}+x_{d 2 i}-\dot{x}_{d 1 i}+k_{1 i} e_{1 i}
$$

Then $\dot{V}_{1 i}$ becomes:

$$
\dot{V}_{1 i}=z_{2 i} e_{1 i}-k_{1 i} e_{1 i}^{2}
$$

To backstep, the system (11) is transformed into the form:

$$
\begin{aligned}
\dot{e}_{1 i}=- & k_{1 i} e_{1 i}+z_{2 i}, \\
\dot{z}_{2 i}=J_{D i} & {\left[\left(G_{D i}-K_{M a}\right)\left(z_{2 i}+\dot{x}_{d 1 i}-k_{1 i} e_{1 i}\right)-M_{Z i}\right]+} \\
& +K_{M b} J_{D i} u_{i}-\ddot{x}_{d 1 i}+k_{1 i} \dot{e}_{1 i} .
\end{aligned}
$$

Now, a new control Lyapunov function $V_{2 i}$ is built by augmenting the control Lyapunov function $V_{1 i}$ obtained in the previous step using a stabilization function. This function penalizes the error between the virtual control and its desired value. So, taking,

$$
V_{2 i}=V_{1 i}+\frac{1}{2} \kappa_{i} z_{2 i}^{2},
$$

as a composite Lyapunov function, we obtain:

$$
\begin{aligned}
& \dot{V}_{2 i}=-k_{1 i} e_{1 i}^{2}+\kappa_{i} z_{2 i}\left[J _ { D i } ( G _ { D i } - K _ { M a } ) \left(z_{2 i}+\dot{x}_{d 1 i}-\right.\right. \\
& \left.\left.-k_{1 i} e_{1 i}\right)-J_{D i} M_{Z i}+K_{M b} J_{D i} u_{i}+\frac{e_{1 i}}{\kappa_{i}}-\ddot{x}_{d 1 i}+k_{1 i} \dot{e}_{1 i}\right] .
\end{aligned}
$$

The choice of control,

$$
\begin{aligned}
u_{i}= & \left(\frac{J_{M}}{K_{M b}}+\frac{J_{G i}}{K_{M b} K_{D\left(e_{1 i}+x_{d 1 i}\right)}^{2}}\right)\left[-\frac{e_{1 i}}{\kappa_{i}}+\ddot{x}_{d 1 i}-\right. \\
& -k_{1 i} \dot{e}_{1 i}-J_{D i}\left(G_{D i}-K_{M a}\right)\left(z_{2 i}+\dot{x}_{d 1 i}-k_{1 i} e_{1 i}\right)- \\
& \left.-J_{D i} M_{Z i}-k_{2 i} z_{2 i}\right],
\end{aligned}
$$

yields,

$$
\dot{V}_{2 i}=-k_{1 i} e_{1 i}^{2}-\kappa_{i} k_{2 i} z_{2 i}^{2},
$$

where $k_{1 i}, k_{2 i}, \kappa_{i}>0$. This implies asymptotical stability according to Lyapunov stability theorem.

\section{EXPERIMENTS}

To evaluate the performance of the tracking control algorithm, different experiments were carried out using a prototype of the Dual Smart Drive. Pulse width modulation technique (PWM) was used to control the voltage delivered to the motor. A 2000-pulse-per-revolution optical encoder was attached to the motor drive to feedback angular position to the controller. The control algorithms were implemented directly in a 486 Processor running real time operating system QNX. The initial conditions for the Dual Smart Drive were $\left(x_{1 i}, x_{2 i}\right)=(-145.4 \mathrm{rad}, 0 \mathrm{rad} / \mathrm{s})$. The time optimal reference trajectories were obtained using the bang-bang control laws:

$$
\begin{array}{llr}
u_{1}^{*}=10.8 \mathrm{~V} & \text { for } & 0 \mathrm{~s}<t \leq 1.165 \mathrm{~s} \\
u_{1}^{*}=-10.8 \mathrm{~V} & \text { for } & 1.165 \mathrm{~s}<t \leq 1.183 \mathrm{~s} \\
u_{2}^{*}=-10.8 \mathrm{~V} & \text { for } & 0 \mathrm{~s}<t \leq 0.5 \mathrm{~s} \\
u_{2}^{*}=10.8 \mathrm{~V} & \text { for } & 0.5 \mathrm{~s}<t \leq 0.518 \mathrm{~s}
\end{array}
$$

Fig. 8-11 show the results obtained using control law (21) with $k_{11}=50, k_{21}=80, \kappa_{1}=1$ for the first working regime of the Dual Smart Drive. Red dotted lines represent the desired values and blue solid lines represent the actual values. In all the experiments a very good tracking performance was obtained with a reasonable control effort. It was possible to appreciate that the first state variable (position) comes very close to the desired level almost immediately, while the second state variable (velocity) experiences lengthier transients. It is also interesting to point out that despite of the tracking errors of the motor, the mobile link tracks 
its reference trajectory almost perfectly, due to the intrinsic properties of the Dual Smart Drive.

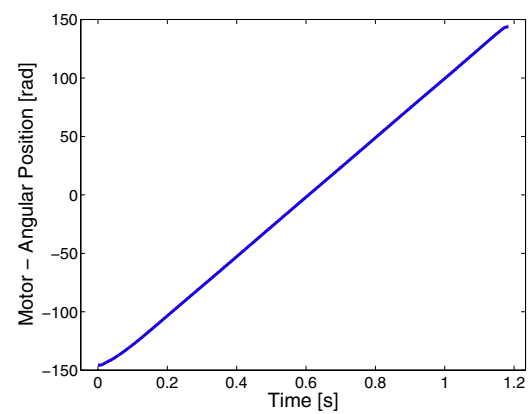

Fig. 8. Time evolution of the angular position of the rotor.

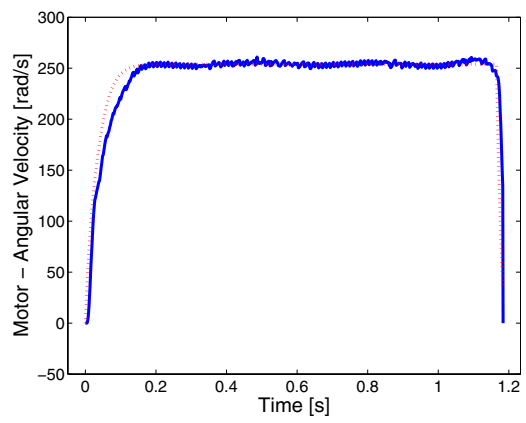

Fig. 9. Time evolution of the angular velocity of the rotor.

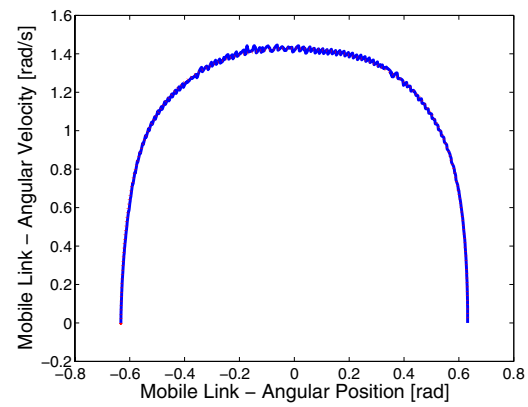

Fig. 10. Reference and actual mobile link trajectories in the phase plane.

\section{CONCLUSIONS}

A nonlinear tracking controller which reflects the idealized dynamics of time optimal control has been presented for the Dual Smart Drive. This controller has been constructed using a backstepping design procedure. Asymptotic stability and the desired tracking performance have been achieved. The limitations of bang-bang control due to modelling inaccuracies and unpredicted disturbances, have been alleviated using backstepping. As modelling and timing accuracies approach perfection, the controllers presented here can approach true time optimal control. Experimental results show the controller effectiveness and demonstrate that the control objectives were accomplished.

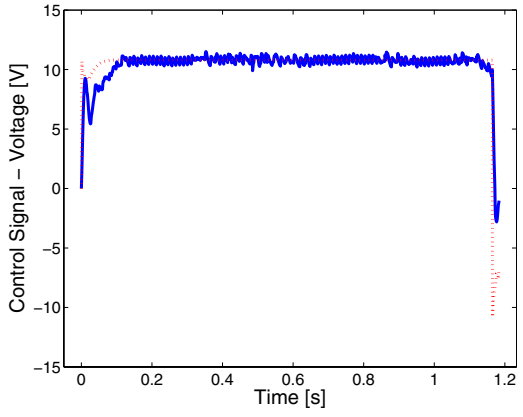

Fig. 11. Behaviour of the control signal.

\section{ACKNOWLEDGMENTS}

R. Fernández would like to acknowledge Spanish Ministry of Education and Science for funding her stay at the Center for Control Engineering and Computation, University of California, Santa Barbara. R. Fernández also would like to thank Professor Petar Kokotović and all the other members of the CCEC lab for a worthwhile and motivating stay.

\section{REFERENCES}

[1] M. Armada, P. González de Santos, M. A. Jiménez and M. Prieto, "Application of CLAWAR Machines," The International Journal of Robotics Research, 2003, Vol. 22, No. 3-4, pp. 251-264.

[2] F. Pfeiffer, K. Löffler and M. Gienger, "Design aspects of walking machines," Proceedings of the $3^{\text {rd }}$ International Conference on Climbing and Walking Robots, Professional Engineering Publishing Limited London, UK, 2000, pp. 17-38.

[3] P. Sardin, M. Rostami and G. Besonet, "An anthropomorphic biped robot: dynamic concepts and technological design," IEEE Transactions on Systems, Man and Cybernetics, Part A, Vol. 28, 1998.

[4] T. Akinfiev, and M. Armada, "Resonance and quasi-resonance drive for start-stop regime," Pergamon, Proc. $6^{\text {th }}$ International Conference MECHATRONICS' 98, Skovde, Sweden, 1998, pp. 91-96.

[5] O. Bruneau, J. P. Louboutin and J. G. Fontaine, "Optimal design of a leg-wheel hybrid robot actuated by a quasi-resonant system," Proceedings of the $3^{\text {rd }}$ International Conference on Climbing and Walking Robots, Professional Engineering Publishing Limited London, UK, 2000, pp. 551-558.

[6] J. Roca, J. Palacin, J. Bradineras and J. M. Iglesias, "Lightweight leg design for a static biped walking robot," Proceedings of the $5^{\text {th }}$ International Conference on Climbing and Walking Robots, Professional Engineering Publishing Limited London, UK, 2002, pp. 383-390.

[7] T. Akinfiev, M. Armada and R. Fernández, "Drive for working element, especially for a walking robot, and the control method," Spanish Patent Office, 2002, Publication Number: 2195792.

[8] M. Athans, and P. L. Falb, Optimal Control, McGraw-Hill Book Company, New York, 1966.

[9] R. Fernández, T. Akinfiev and M. Armada, "Dual smart drive: analytical solution, simulation and experimental results," Proceedings of the $6^{\text {th }}$ International Conference on Climbing and Walking Robots and the Support Technologies for Mobile Machines, Professional Engineering Publishing Limited London, UK, 2003, pp. 309-318.

[10] F. Song, and S. M. Smith, "Design of sliding mode fuzzy controllers for an autonomous underwater vehicle without system model," Oceans'2000 MTS/IEEE, pp. 835-840, 2000.

[11] P. H. Meckl, and W. Seering, "Active damping in a three-axis robotic manipulator," A.S.M.E. Journal of Vibration, Acoustic, Stress, and Reliability in Design, 107, pp.38-46, 1985.

[12] P. V. Kokotović, "The Joy of Feedback: Nonlinear and Adaptive," IEEE Contr. Sys. Mag., vol. 12, pp. 7-17, 1992.

[13] M. Krstić, I. Kanellakopoulos and P. V. Kokotović, Nonlinear and Adaptive Control Design, New York: Wiley, 1995.

[14] H. K. Khalil, Nonlinear Systems, New York: Prentice Hall, 2002. 